\title{
A parallel resolvent method for solving a system of nonlinear mixed variational inequalities
}

\author{
Ke Guo*, Ya Jiang and Shi-Qiang Feng
}

\section{"Correspondence:} robertjo@126.com College of Mathematics and Information, China West Normal University, Nanchong, Sichuan 637009, China

\begin{abstract}
In this paper, we introduce a system of generalized nonlinear mixed variational inequalities and obtain the approximate solvability by using the resolvent parallel technique. Our results may be viewed as an extension and improvement of the previously known results for variational inequalities.
\end{abstract}

Keywords: resolvent operator; parallel projection; relaxed cocoercive; generalized nonlinear mixed variational inequalities

\section{Introduction and preliminaries}

Variational inequality theory, which was introduced by Stampacchia [1] in 1964, has been witnessed as an interesting branch of mathematical and engineering sciences with a wide range of applications in industry, finance, economics and pure and applied sciences. In 2001, Verma [2] introduced a new system of strongly monotonic variational inequalities and studied the approximation solvability of the system based on the application of a projection method. The main and basic idea in this technique is to establish the equivalence between variational inequalities and fixed point problems. This alternative equivalence has been used to develop several projection iterative methods for solving variational inequalities and related optimization problems. Several extensions and generalizations of the system of strongly monotonic variational inequalities have been considered by many authors [3-12]. Inspired and motivated by research in this area, we introduce a system of generalized nonlinear mixed variational inequalities problem involving two different nonlinear operators. It is well known that if the nonlinear term in the mixed variational inequality is a proper, convex, and lower semicontinuous, then one can establish the equivalence between the mixed variational inequality and the fixed point problem. Using the parallel algorithm considered in [12], we suggest and analyze a parallel iterative method for solving this system. Our result may be viewed as an extension and improvement of the recent results.

Let $\mathcal{H}$ be a real Hilbert space whose inner product and norm are denoted by $\langle\cdot, \cdot\rangle$ and $\|\cdot\|$, respectively. Let $K$ be a nonempty closed convex subset of $\mathcal{H}$. Let $T_{1}, T_{2}: K \times K \rightarrow \mathcal{H}$ be two nonlinear operators. Let $\varphi_{1}, \varphi_{2}: \mathcal{H} \rightarrow \mathbb{R} \cup\{+\infty\}$ be proper convex lower semicontinuous functions on $\mathcal{H}$. We consider a system of generalized nonlinear mixed varia- 
tional inequalities (abbreviated as SMNVI) as follows: Find $\left(x^{*}, y^{*}\right) \in K \times K$ such that

$$
\left\{\begin{array}{l}
\left\langle\rho T_{1}\left(y^{*}, x^{*}\right)+g\left(x^{*}\right)-g\left(y^{*}\right), g(x)-g\left(x^{*}\right)\right\rangle+\varphi_{1}(g(x))-\varphi_{1}\left(g\left(x^{*}\right)\right) \\
\geq 0, \quad \forall g(x) \in K, \\
\left\langle\eta T_{2}\left(x^{*}, y^{*}\right)+g\left(y^{*}\right)-g\left(x^{*}\right), g(x)-g\left(x^{*}\right)\right\rangle+\varphi_{2}(g(x))-\varphi_{2}\left(g\left(y^{*}\right)\right) \\
\geq 0, \quad \forall g(x) \in K,
\end{array}\right.
$$

where $g: K \rightarrow K$ is a mapping and $\rho, \eta>0$.

Note that if $\varphi_{1}=\varphi_{2}=\delta_{K}$, and $g=I$, where $I$ is the identity operator, $\delta_{K}$ is the indicator function of $K$ defined by

$$
\delta_{K}(x)= \begin{cases}0 & \text { if } x \in K \\ +\infty & \text { otherwise }\end{cases}
$$

then problem (1.1) reduces to the following system of nonlinear variational inequalities (SNVI) considered in [3] of finding $\left(x^{*}, y^{*}\right) \in K \times K$ such that

$$
\begin{cases}\left\langle\rho T_{1}\left(y^{*}, x^{*}\right)+x^{*}-y^{*}, x-x^{*}\right\rangle \geq 0, & \forall x \in K, \\ \left\langle\eta T_{2}\left(x^{*}, y^{*}\right)+y^{*}-x^{*}, x-x^{*}\right\rangle \geq 0, & \forall x \in K .\end{cases}
$$

If $T_{1}=T_{2}=T$ and $g=I$, where $I$ is the identity operator, then problem (1.1) is equivalent to the following system of nonlinear mixed variational inequalities (SNVI) considered in $[7,8]$ of finding $\left(x^{*}, y^{*}\right) \in K \times K$ such that

$$
\begin{cases}\left\langle\rho T\left(y^{*}, x^{*}\right)+x^{*}-y^{*}, x-x^{*}\right\rangle+\varphi_{1}(x)-\varphi_{1}\left(x^{*}\right) \geq 0, & \forall x \in K, \\ \left\langle\eta T\left(x^{*}, y^{*}\right)+y^{*}-x^{*}, x-x^{*}\right\rangle+\varphi_{2}(x)-\varphi_{2}\left(y^{*}\right) \geq 0, & \forall x \in K .\end{cases}
$$

If $\varphi_{1}=\varphi_{2}=\delta_{K}$ and $T_{1}, T_{2}: K \rightarrow \mathcal{H}$ are univariate mappings, then problem (1.1) is reduced to the following system of nonlinear variational inequalities (SNVI) considered in [12] of finding $\left(x^{*}, y^{*}\right) \in K \times K$ such that

$$
\begin{cases}\left\langle\rho T_{1}\left(y^{*}\right)+g\left(x^{*}\right)-g\left(y^{*}\right), g(x)-g\left(x^{*}\right)\right\rangle \geq 0, & \forall g(x) \in K, \\ \left\langle\eta T_{2}\left(x^{*}\right)+g\left(y^{*}\right)-g\left(x^{*}\right), g(x)-g\left(x^{*}\right)\right\rangle \geq 0, & \forall g(x) \in K,\end{cases}
$$

where $g: K \rightarrow K$ is a mapping.

If $T_{1}=T_{2}=T, g=I$ and $\varphi_{1}=\varphi_{2}=\delta_{K}$, where $T$ is a univariate mapping defined by $T$ : $K \rightarrow \mathcal{H}$, then problem (1.1) reduces to the following system of variational inequalities (SVI) considered in [2] of finding $\left(x^{*}, y^{*}\right) \in K \times K$ such that

$$
\begin{cases}\left\langle\rho T\left(y^{*}\right)+x^{*}-y^{*}, x-x^{*}\right\rangle \geq 0, & \forall x \in K, \\ \left\langle\eta T\left(x^{*}\right)+y^{*}-x^{*}, x-x^{*}\right\rangle \geq 0, & \forall x \in K .\end{cases}
$$

We also need the following well-known results. 
Definition 1.1 Define the norm $\|\cdot\|$ on $\mathcal{H} \times \mathcal{H}$ by

$$
\|(u, v)\|=\|u\|+\|v\|, \quad \forall(u, v) \in \mathcal{H} \times \mathcal{H} .
$$

Definition 1.2 For any maximal monotone operator $T$, the resolvent operator associated with $T$, for any $\lambda>0$, is defined by

$$
J_{T}^{\lambda}(u)=(I+\lambda T)^{-1}(u), \quad \forall u \in \mathcal{H} .
$$

Remark 1.1 It is well known that the subdifferential $\partial \varphi$ of a proper convex lower semicontinuous function $\varphi: \mathcal{H} \rightarrow \mathbb{R} \cup\{+\infty\}$ is a maximal monotone operator. We can define its resolvent operator by

$$
J_{\varphi}^{\lambda}(u)=(I+\lambda \partial \varphi)^{-1}(u), \quad \forall u \in \mathcal{H},
$$

where $\lambda>0$ and $J_{\varphi}$ is defined everywhere.

Lemma 1.1 [13] For a given $u, z \in \mathcal{H}$ satisfies the inequality

$$
\langle u-z, x-u\rangle+\lambda \varphi(x)-\lambda \varphi(u) \geq 0, \quad \forall x \in \mathcal{H},
$$

if and only if $u=J_{\varphi}^{\lambda}(z)$, where $J_{\varphi}^{\lambda}(u)=(I+\lambda \partial \varphi)^{-1}(u)$ is the resolvent operator and $\lambda>0$.

If $\varphi$ is the indicator function of a closed convex set $K \subseteq \mathcal{H}$, then the resolvent operator $J_{\varphi}^{\lambda}(\cdot)$ reduces to the projection operator $P_{K}(\cdot)$. It is well known that $J_{\varphi}^{\lambda}$ is nonexpansive, i.e.,

$$
\left\|J_{\varphi}^{\lambda}(u)-J_{\varphi}^{\lambda}(v)\right\| \leq\|u-v\|, \quad \forall u, v \in \mathcal{H}
$$

Based on Lemma 1.1, similar to that in [8] and [7], the following statement gives equivalent characterization of problem (1.1).

Lemma 1.2 Problem (1.1) is equivalent to finding $\left(x^{*}, y^{*}\right) \in K \times K$ such that

$$
\left\{\begin{array}{l}
g\left(x^{*}\right)=J_{\varphi_{1}}^{1}\left[g\left(y^{*}\right)-\rho T_{1}\left(y^{*}, x^{*}\right)\right], \\
g\left(y^{*}\right)=J_{\varphi_{2}}^{1}\left[g\left(x^{*}\right)-\eta T_{2}\left(x^{*}, y^{*}\right)\right],
\end{array}\right.
$$

where $J_{\varphi_{i}}^{1}=\left(I+\partial \varphi_{i}\right)^{-1}, i=1,2$.

Proof Suppose that $\left(x^{*}, y^{*}\right) \in K \times K$ is a solution of the following generalized nonlinear mixed variational inequalities (abbreviated as SNMVI):

$$
\left\{\begin{array}{l}
\left\langle\rho T_{1}\left(y^{*}, x^{*}\right)+g\left(x^{*}\right)-g\left(y^{*}\right), g(x)-g\left(x^{*}\right)\right\rangle+\rho^{\prime} \varphi_{1}(g(x))-\rho^{\prime} \varphi_{1}\left(g\left(x^{*}\right)\right) \\
\geq 0, \quad \forall g(x) \in K, \\
\left\langle\eta T_{2}\left(x^{*}, y^{*}\right)+g\left(y^{*}\right)-g\left(x^{*}\right), g(x)-g\left(x^{*}\right)\right\rangle+\eta^{\prime} \varphi_{2}(g(x))-\eta^{\prime} \varphi_{2}\left(g\left(y^{*}\right)\right) \\
\geq 0, \quad \forall g(x) \in K,
\end{array}\right.
$$


where $g: K \rightarrow K$ is a mapping and $\rho>0, \rho^{\prime}>0, \eta>0, \eta^{\prime}>0$. Using Lemma 1.1, we can easily show that problem (1.7) is equivalent to

$$
\left\{\begin{array}{l}
g\left(x^{*}\right)=J_{\varphi_{1}}^{\rho^{\prime}}\left[g\left(y^{*}\right)-\rho T_{1}\left(y^{*}, x^{*}\right)\right], \\
g\left(y^{*}\right)=J_{\varphi_{2}}^{\eta^{\prime}}\left[g\left(x^{*}\right)-\eta T_{2}\left(x^{*}, y^{*}\right)\right],
\end{array}\right.
$$

where $J_{\varphi_{1}}^{\rho^{\prime}}=\left(I+\rho^{\prime} \partial \varphi_{1}\right)^{-1}, J_{\varphi_{2}}^{\eta^{\prime}}=\left(I+\eta^{\prime} \partial \varphi_{2}\right)^{-1}$. Let $\rho^{\prime}=\eta^{\prime}=1$. Then problem (1.7) reduces to problem (1.1) and $J_{\varphi_{i}}^{1}=\left(I+\partial \varphi_{i}\right)^{-1}, i=1,2$. This completes the proof.

Remark 1.2 If $T_{1}=T_{2}=T$ and $g=I$, where $I$ is the identity operator, then Lemma 1.2 reduces to Lemma 1.2 in [7].

Definition 1.3 A mapping $T: K \times K \rightarrow \mathcal{H}$ is said to be

(1) relaxed $g-(\gamma, r)$-cocoercive if there exist constants $\gamma>0$ and $r>0$ such that for all $x, y \in K$,

$$
\langle T(x, u)-T(y, v), g(x)-g(y)\rangle \geq(-\gamma)\|T(x, u)-T(y, v)\|^{2}+r\|g(x)-g(y)\|^{2}, \quad \forall u, v \in K
$$

(2) $g$ - $\mu$-Lipschitz continuous in the first variable if there exists a constant $\mu>0$ such that for all $x, y \in K$,

$$
\|T(x, u)-T(y, v)\| \leq \mu\|g(x)-g(y)\|, \quad \forall u, v \in K .
$$

Remark 1.3 If $T$ is a univariate mapping and $g=I$, where $I$ is the identity operator, then Definition 1.3 reduces to the standard definition of relaxed $(\gamma, r)$-cocoercive and Lipschitz continuous, respectively.

Definition 1.4 A mapping $g: K \rightarrow \mathcal{H}$ is said to be $\alpha$-expansive if there exists a constant $\alpha>0$ such that for all $x, y \in \mathcal{H}$,

$$
\|g(x)-g(y)\| \geq \alpha\|x-y\| .
$$

Lemma 1.3 [14] Suppose that $\left\{\delta_{n}\right\}$ is a nonnegative sequence satisfying the following inequality:

$$
\delta_{n+1} \leq\left(1-\lambda_{n}\right) \delta_{n}+\sigma_{n}, \quad \forall n \geq n_{0},
$$

where $n_{0}$ is a nonnegative number, $\lambda_{n} \in[0,1]$ with $\sum_{n=0}^{\infty} \lambda_{n}=\infty$, and $\sigma_{n}=o\left(\lambda_{n}\right)$. Then $\lim _{n \rightarrow \infty} \delta_{n}=0$.

\section{Algorithms}

In this section, we suggest a parallel algorithm associated with the resolvent operator for solving the system of SNMVI. Our results extend and improve the corresponding results in $[2,3,7,11,12]$. In fact, using Lemma 1.2, we suggest the following iterative method for solving problem (1.1). 
Algorithm 2.1 For arbitrarily chosen initial points $x_{0}, y_{0} \in K$ (and $\left.g\left(x_{0}\right), g\left(y_{0}\right) \in K\right)$, compute the sequences $\left\{x_{n}\right\}$ and $\left\{y_{n}\right\}$ such that

$$
\left\{\begin{array}{l}
g\left(x_{n+1}\right)=\left(1-\alpha_{n}\right) g\left(x_{n}\right)+\alpha_{n} J_{\varphi_{1}}^{1}\left[g\left(y_{n}\right)-\rho T_{1}\left(y_{n}, x_{n}\right)\right], \\
g\left(y_{n+1}\right)=\left(1-\beta_{n}\right) g\left(y_{n}\right)+\beta_{n} J_{\varphi_{2}}^{1}\left[g\left(x_{n}\right)-\eta T_{2}\left(x_{n}, y_{n}\right)\right],
\end{array}\right.
$$

where $J_{\varphi_{i}}^{1}=\left(I+\partial \varphi_{i}\right)^{-1}, i=1,2$, is the resolvent operator, $\rho, \eta>0, \alpha_{n} \in[0,1]$ and $\beta_{n} \in[0,1]$ for all $n \geq 0$.

As reported in [12], one of the attractive features of Algorithm 2.1 is that it is suitable for implementing on two different processor computers. In other words, $x_{n+1}$ and $y_{n+1}$ are solved in parallel, and Algorithm 2.1 is the so-called parallel resolvent method. We refer the interested reader to papers [15-17] and references therein for more examples and ideas of parallel iterative methods.

If $\varphi_{1}=\varphi_{2}=\delta_{K}$, and $g=I, \delta_{K}$ is the indicator function of $K$, then Algorithm 2.1 reduces to the following algorithm.

Algorithm 2.2 For arbitrarily chosen initial points $x_{0}, y_{0} \in K$, compute the sequences $\left\{x_{n}\right\}$ and $\left\{y_{n}\right\}$ such that

$$
\left\{\begin{array}{l}
x_{n+1}=\left(1-\alpha_{n}\right) x_{n}+\alpha_{n} P_{K}\left[y_{n}-\rho T_{1}\left(y_{n}, x_{n}\right)\right], \\
y_{n+1}=\left(1-\beta_{n}\right) y_{n}+\beta_{n} P_{K}\left[x_{n}-\eta T_{2}\left(x_{n}, y_{n}\right)\right],
\end{array}\right.
$$

where $\rho, \eta>0, \alpha_{n} \in[0,1]$ and $\beta_{n} \in[0,1]$ for all $n \geq 0$.

If $T_{1}=T_{2}=T$ and $g=I$, then Algorithm 2.1 reduces to the following algorithm.

Algorithm 2.3 For arbitrarily chosen initial points $x_{0}, y_{0} \in K$, compute the sequences $\left\{x_{n}\right\}$ and $\left\{y_{n}\right\}$ such that

$$
\left\{\begin{array}{l}
x_{n+1}=\left(1-\alpha_{n}\right) x_{n}+\alpha_{n} J_{\varphi_{1}}^{1}\left[y_{n}-\rho T\left(y_{n}, x_{n}\right)\right], \\
y_{n+1}=\left(1-\beta_{n}\right) y_{n}+\beta_{n} J_{\varphi_{2}}^{1}\left[x_{n}-\eta T\left(x_{n}, y_{n}\right)\right],
\end{array}\right.
$$

where $J_{\varphi_{i}}^{1}=\left(I+\partial \varphi_{i}\right)^{-1}, i=1,2$, is the resolvent operator, $\rho, \eta>0, \alpha_{n} \in[0,1]$ and $\beta_{n} \in[0,1]$ for all $n \geq 0$.

If $\varphi_{1}=\varphi_{2}=\delta_{K}$ and $T_{1}, T_{2}: K \rightarrow \mathcal{H}$ are univariate mappings, then Algorithm 2.1 reduces to the following algorithm.

Algorithm 2.4 For arbitrarily chosen initial points $x_{0}, y_{0} \in K$ (and $\left.g\left(x_{0}\right), g\left(y_{0}\right) \in K\right)$, compute the sequences $\left\{x_{n}\right\}$ and $\left\{y_{n}\right\}$ such that

$$
\left\{\begin{array}{l}
g\left(x_{n+1}\right)=\left(1-\alpha_{n}\right) g\left(x_{n}\right)+\alpha_{n} P_{K}\left[g\left(y_{n}\right)-\rho T_{1}\left(y_{n}\right)\right], \\
g\left(y_{n+1}\right)=\left(1-\beta_{n}\right) g\left(y_{n}\right)+\beta_{n} P_{K}\left[g\left(x_{n}\right)-\eta T_{2}\left(x_{n}\right)\right],
\end{array}\right.
$$

where $\rho, \eta>0, \alpha_{n} \in[0,1]$ and $\beta_{n} \in[0,1]$ for all $n \geq 0$. 
If $T_{1}=T_{2}=T, g=I$ and $\varphi_{1}=\varphi_{2}=\delta_{K}$, where $T$ is a univariate mapping defined by $T$ : $K \rightarrow \mathcal{H}$, then Algorithm 2.1 reduces to the following algorithm.

Algorithm 2.5 For arbitrarily chosen initial points $x_{0}, y_{0} \in K\left(\right.$ and $g\left(x_{0}\right), g\left(y_{0}\right) \in K$ ), compute the sequences $\left\{x_{n}\right\}$ and $\left\{y_{n}\right\}$ such that

$$
\left\{\begin{array}{l}
x_{n+1}=\left(1-\alpha_{n}\right) x_{n}+\alpha_{n} P_{K}\left[y_{n}-\rho T\left(y_{n}\right)\right], \\
y_{n+1}=\left(1-\beta_{n}\right) y_{n}+\beta_{n} P_{K}\left[x_{n}-\eta T\left(x_{n}\right)\right],
\end{array}\right.
$$

where $\rho, \eta>0, \alpha_{n} \in[0,1]$ and $\beta_{n} \in[0,1]$ for all $n \geq 0$.

\section{Main results}

In this section, based on Algorithm 2.1, we now present the approximation solvability of problem (1.1) involving relaxed $g-(\gamma, r)$-cocoercive and $g-\mu$-Lipschitz continuous in the first variable mappings in Hilbert settings.

Theorem 3.1 Let $\mathcal{H}$ be a real Hilbert space. Let $K$ be a nonempty closed convex subset of $\mathcal{H}$, and let $T_{i}: K \times K \rightarrow \mathcal{H}$ be relaxed $g-\left(\gamma_{i}, r_{i}\right)$-cocoercive and $g-\mu_{i}$-Lipschitz continuous in the first variable for $i=1,2$. Let $g: K \rightarrow K$ be an $\alpha$-expansive mapping. Suppose that $\left(x^{*}, y^{*}\right) \in$ $K \times K$ is the unique solution to problem (1.1) and $\left\{x_{n}\right\},\left\{y_{n}\right\}$ are generated by Algorithm 2.1. If $\left\{\alpha_{n}\right\}$ and $\left\{\beta_{n}\right\}$ are two sequences in $[0,1]$ satisfying the following conditions:

(i) $\alpha_{n}-\theta_{2} \beta_{n} \geq 0$ and $\beta_{n}-\theta_{1} \alpha_{n} \geq 0$ such that $\sum_{n=0}^{\infty} \alpha_{n}-\theta_{2} \beta_{n}=\infty$, $\sum_{n=0}^{\infty} \beta_{n}-\theta_{1} \alpha_{n}=\infty$

(ii) $\theta_{1}=\sqrt{1+2 \rho \gamma_{1} \mu_{1}^{2}-2 \rho r_{1}+\rho^{2} \mu_{1}}$ such that $0<\theta_{1}<1$,

(iii) $\theta_{2}=\sqrt{1+2 \eta \gamma_{2} \mu_{2}^{2}-2 \eta r_{2}+\eta^{2} \mu_{2}}$ such that $0<\theta_{2}<1$,

then the sequences $\left\{x_{n}\right\}$ and $\left\{y_{n}\right\}$ converge to $x^{*}$ and $y^{*}$, respectively.

Proof Since $\left(x^{*}, y^{*}\right) \in K \times K$ is the unique solution to problem (1.1), from Lemma 1.2 it follows that

$$
\left\{\begin{array}{l}
g\left(x^{*}\right)=J_{\varphi_{1}}^{1}\left[g\left(y^{*}\right)-\rho T_{1}\left(y^{*}, x^{*}\right)\right], \\
g\left(y^{*}\right)=J_{\varphi_{2}}^{1}\left[g\left(x^{*}\right)-\eta T_{2}\left(x^{*}, y^{*}\right)\right] .
\end{array}\right.
$$

We first evaluate $\left\|g\left(x_{n+1}\right)-g\left(x^{*}\right)\right\|$ for all $n \geq 0$. From (2.1) and the nonexpansive property of the resolvent operator, we have

$$
\begin{aligned}
& \left\|g\left(x_{n+1}\right)-g\left(x^{*}\right)\right\| \\
& =\|\left(1-\alpha_{n}\right) g\left(x_{n}\right)+\alpha_{n} J_{\varphi_{1}}^{1}\left[g\left(y_{n}\right)-\rho T_{1}\left(y_{n}, x_{n}\right)\right] \\
& \quad-\left(1-\alpha_{n}\right) g\left(x^{*}\right)-\alpha_{n} J_{\varphi_{1}}^{1}\left[g\left(y^{*}\right)-\rho T_{1}\left(y^{*}, x^{*}\right)\right] \| \\
& \quad \leq \quad\left(1-\alpha_{n}\right)\left\|g\left(x_{n}\right)-g\left(x^{*}\right)\right\| \\
& \quad+\alpha_{n}\left\|J_{\varphi_{1}}^{1}\left[g\left(y_{n}\right)-\rho T_{1}\left(y_{n}, x_{n}\right)\right]-J_{\varphi_{1}}^{1}\left[g\left(y^{*}\right)-\rho T_{1}\left(y^{*}, x^{*}\right)\right]\right\| \\
& \leq\left(1-\alpha_{n}\right)\left\|g\left(x_{n}\right)-g\left(x^{*}\right)\right\|+\alpha_{n}\left\|g\left(y_{n}\right)-g\left(y^{*}\right)-\rho\left(T_{1}\left(y_{n}, x_{n}\right)-T_{1}\left(y^{*}, x^{*}\right)\right)\right\| .
\end{aligned}
$$


Notice that $T_{1}$ is relaxed $g$ - $\left(\gamma_{1}, r_{1}\right)$-cocoercive and $g$ - $\mu_{1}$-Lipschitz continuous in the first variable. Then we have

$$
\begin{aligned}
&\left\|g\left(y_{n}\right)-g\left(y^{*}\right)-\rho\left(T_{1}\left(y_{n}, x_{n}\right)-T_{1}\left(y^{*}, x^{*}\right)\right)\right\|^{2} \\
&=\left\|g\left(y_{n}\right)-g\left(y^{*}\right)\right\|^{2}-2 \rho\left(g\left(y_{n}\right)-g\left(y^{*}\right), T_{1}\left(y_{n}, x_{n}\right)-T_{1}\left(y^{*}, x^{*}\right)\right\rangle \\
&+\rho^{2}\left\|T_{1}\left(y_{n}, x_{n}\right)-T_{1}\left(y^{*}, x^{*}\right)\right\|^{2} \\
& \leq\left\|g\left(y_{n}\right)-g\left(y^{*}\right)\right\|^{2}+2 \rho \gamma_{1}\left\|T_{1}\left(y_{n}, x_{n}\right)-T_{1}\left(y^{*}, x^{*}\right)\right\|^{2} \\
& \quad-2 \rho r_{1}\left\|g\left(y_{n}\right)-g\left(y^{*}\right)\right\|^{2}+\rho^{2}\left\|T_{1}\left(y_{n}, x_{n}\right)-T_{1}\left(y^{*}, x^{*}\right)\right\|^{2} \\
& \leq \theta^{2}\left\|g\left(y_{n}\right)-g\left(y^{*}\right)\right\|^{2},
\end{aligned}
$$

where $\theta_{1}=\sqrt{1+2 \rho \gamma_{1} \mu_{1}^{2}-2 \rho r_{1}+\rho^{2} \mu_{1}}<1$ in view of assumption (ii). Substituting (3.3) into (3.2), we have

$$
\left\|g\left(x_{n+1}\right)-g\left(x^{*}\right)\right\| \leq\left(1-\alpha_{n}\right)\left\|g\left(x_{n}\right)-g\left(x^{*}\right)\right\|+\alpha_{n} \theta_{1}\left\|g\left(y_{n}\right)-g\left(y^{*}\right)\right\| .
$$

Similarly, since $T_{2}$ is relaxed $g$ - $\left(\gamma_{2}, r_{2}\right)$-cocoercive and $g$ - $\mu_{2}$-Lipschitz continuous in the first variable, we have

$$
\left\|g\left(y_{n+1}\right)-g\left(y^{*}\right)\right\| \leq\left(1-\beta_{n}\right)\left\|g\left(y_{n}\right)-g\left(y^{*}\right)\right\|+\beta_{n} \theta_{2}\left\|g\left(x_{n}\right)-g\left(x^{*}\right)\right\|,
$$

where $\theta_{2}=\sqrt{1+2 \eta \gamma_{2} \mu_{2}^{2}-2 \eta r_{2}+\eta^{2} \mu_{2}}<1$ in view of assumption (iii). It follows from (3.4) and (3.5) that

$$
\begin{aligned}
& \left\|\left(g\left(x_{n+1}\right), g\left(y_{n+1}\right)\right)-\left(g\left(x^{*}\right), g\left(y^{*}\right)\right)\right\| \\
& \quad \leq\left[1-\left(\alpha_{n}-\theta_{2} \beta_{n}\right)\right]\left\|g\left(x_{n}\right)-g\left(x^{*}\right)\right\|+\left[1-\left(\beta_{n}-\theta_{1} \alpha_{n}\right)\right]\left\|g\left(y_{n}\right)-g\left(y^{*}\right)\right\| \\
& \quad=\max \left\{w_{1 n}, w_{2 n}\right\}\left(\left\|\left(g\left(x_{n}\right), g\left(y_{n}\right)\right)-\left(g\left(x^{*}\right), g\left(y^{*}\right)\right)\right\|\right),
\end{aligned}
$$

where $w_{1 n}=1-\left(\alpha_{n}-\theta_{2} \beta_{n}\right)$ and $w_{2 n}=1-\left(\beta_{n}-\theta_{1} \alpha_{n}\right)$.

From assumption (i) and Lemma 1.3, we can obtain

$$
\lim _{n \rightarrow \infty}\left\|\left(g\left(x_{n+1}\right), g\left(y_{n+1}\right)\right)-\left(g\left(x^{*}\right), g\left(y^{*}\right)\right)\right\|=0,
$$

and so

$$
\lim _{n \rightarrow \infty}\left\|g\left(x_{n+1}\right)-g\left(x^{*}\right)\right\|=0 \quad \text { and } \quad \lim _{n \rightarrow \infty}\left\|g\left(y_{n+1}\right)-g\left(y^{*}\right)\right\|=0,
$$

which implies that sequences $\left\{g\left(x_{n}\right)\right\}$ and $\left\{g\left(y_{n}\right)\right\}$ converge to $g\left(x^{*}\right)$ and $g\left(y^{*}\right)$, respectively. Since $g$ is $\alpha$-expansive, it follows that $\left\{x_{n}\right\}$ and $\left\{y_{n}\right\}$ converge to $x^{*}$ and $y^{*}$, respectively. This completes the proof.

The following theorems can be obtained from Theorem 3.1 immediately.

Theorem 3.2 [3] Let $\mathcal{H}$ be a real Hilbert space. Let $K$ be a nonempty closed convex subset of $\mathcal{H}$, and let $T_{i}: K \times K \rightarrow \mathcal{H}$ be relaxed $\left(\gamma_{i}, r_{i}\right)$-cocoercive and $\mu_{i}$-Lipschitz continuous in 
the first variable for $i=1,2$. Suppose that $\left(x^{*}, y^{*}\right) \in K \times K$ is the unique solution to problem (1.2) and $\left\{x_{n}\right\},\left\{y_{n}\right\}$ are generated by Algorithm 2.2. If $\left\{\alpha_{n}\right\}$ and $\left\{\beta_{n}\right\}$ are two sequences in $[0,1]$ satisfying the following conditions:

(1) $\alpha_{n}-\theta_{2} \beta_{n} \geq 0$ and $\beta_{n}-\theta_{1} \alpha_{n} \geq 0$ such that $\sum_{n=0}^{\infty} \alpha_{n}-\theta_{2} \beta_{n}=\infty, \sum_{n=0}^{\infty} \beta_{n}-\theta_{1} \alpha_{n}=\infty$,

(2) $\theta_{1}=\sqrt{1+2 \rho \gamma_{1} \mu_{1}^{2}-2 \rho r_{1}+\rho^{2} \mu_{1}}$ such that $0<\theta_{1}<1$,

(3) $\theta_{2}=\sqrt{1+2 \eta \gamma_{2} \mu_{2}^{2}-2 \eta r_{2}+\eta^{2} \mu_{2}}$ such that $0<\theta_{2}<1$,

then the sequences $\left\{x_{n}\right\}$ and $\left\{y_{n}\right\}$ converge to $x^{*}$ and $y^{*}$, respectively.

Theorem 3.3 [12] Let $\mathcal{H}$ be a real Hilbert space. Let $K$ be a nonempty closed convex subset of $\mathcal{H}$, and let $T_{i}: K \rightarrow \mathcal{H}$ be relaxed $g-\left(\gamma_{i}, r_{i}\right)$-cocoercive and $g-\mu_{i}$-Lipschitz continuous for $i=1,2$. Let $g: K \rightarrow K$ be an $\alpha$-expansive mapping. Suppose that $\left(x^{*}, y^{*}\right) \in K \times K$ is the unique solution to problem (1.4) and $\left\{x_{n}\right\},\left\{y_{n}\right\}$ are generated by Algorithm 2.4. If $\left\{\alpha_{n}\right\}$ and $\left\{\beta_{n}\right\}$ are two sequences in $[0,1]$ satisfying the following conditions:

(1) $0 \leq \alpha_{n}, \beta_{n} \leq 1, \alpha_{n}-\theta_{2} \beta_{n} \geq 0$ and $\beta_{n}-\theta_{1} \alpha_{n} \geq 0$ such that $\sum_{n=0}^{\infty} \alpha_{n}-\theta_{2} \beta_{n}=\infty$, $\sum_{n=0}^{\infty} \beta_{n}-\theta_{1} \alpha_{n}=\infty$

(2) $\theta_{1}=\sqrt{1+2 \rho \gamma_{1} \mu_{1}^{2}-2 \rho r_{1}+\rho^{2} \mu_{1}}$ such that $0<\theta_{1}<1$,

(3) $\theta_{2}=\sqrt{1+2 \eta \gamma_{2} \mu_{2}^{2}-2 \eta r_{2}+\eta^{2} \mu_{2}}$ such that $0<\theta_{2}<1$,

then the sequences $\left\{x_{n}\right\}$ and $\left\{y_{n}\right\}$ converge to $x^{*}$ and $y^{*}$, respectively.

Competing interests

The authors declare that they have no competing interests.

Authors' contributions

All authors contributed significantly in writing the paper. All authors read and approved the final manuscript.

\section{Acknowledgements}

This work was supported by the Natural Science Foundation of China $(60804065,11371015)$, the Key Project of Chinese Ministry of Education (211163), Sichuan Youth Science and Technology Foundation (2012JQ0032).

Received: 19 December 2012 Accepted: 18 October 2013 Published: 08 Nov 2013

\section{References}

1. Stampacchia, G: Formes bilineaires coercitives sur les ensembles convexes. C. R. Math. Acad. Sci. Paris 258, 4413-4416 (1964)

2. Verma, RU: Projection methods, algorithms and a new system of nonlinear variational inequalities. Comput. Math Appl. 41, 1025-1031 (2001)

3. Chang, SS, Joseph Lee, HW, Chan, CK: Generalized system for relaxed cocoercive variational inequalities in Hilbert spaces. Appl. Math. Lett. 20, 329-334 (2007)

4. Fang, YP, Huang, NJ, Cao, YJ, Kang, SM: Stable iterative algorithms for a class of general nonlinear variational inequalities. Adv. Nonlinear Var. Inequal. 5(2), 1-9 (2002)

5. Fang, YP, Huang, NJ: H-Monotone operator and resolvent operator technique for variational inclusions. Appl. Math. Comput. 145(2-3), 795-803 (2003)

6. Fang, YP, Huang, NJ: H-Accretive operators and resolvent operator technique for solving variational inclusions in Banach spaces. Appl. Math. Lett. 17(6), 647-653 (2004)

7. He, Z, Gu, F: Generalized system for relaxed cocoercive mixed variational inequalities in Hilbert spaces. Appl. Math. Comput. 214, 26-30 (2009)

8. Narin, P: A resolvent operator technique for approximate solving of generalized system mixed variational inequality and fixed point problems. Appl. Math. Lett. 23, 440-445 (2010)

9. Nie, NH, Liu, Z, Kim, KH, Kang, SM: A system of nonlinear variational inequalities involving strong monotone and pseudocontractive mappings. Adv. Nonlinear Var. Inequal. 6, 91-99 (2003)

10. Verma, RU: Generalized system for relaxed cocoercive variational inequalities and its projection methods. J. Optim. Theory Appl. 121, 203-210 (2004)

11. Verma, RU: General convergence analysis for two-step projection methods and applications to variational problems Appl. Math. Lett. 18(11), 1286-1292 (2005)

12. Yang, HJ, Zhou, L, Li, QG: A parallel projection method for a system of nonlinear variational inequalities. Appl. Math. Comput. 217, 1971-1975 (2010)

13. Brezis, $\mathrm{H}$ : Opérateurs maximaux monotone et semi-groupes de contractions dans les espaces de Hilbert. North-Holland Mathematics Studies, vol. 5. Notas de matematica, vol. 50. North-Holland, Amsterdam (1973)

14. Weng, XL: Fixed point iteration for local strictly pseudocontractive mappings. Proc. Am. Math. Soc. 113, 727-731 (1991) 
15. Bertsekas, D, Tsitsiklis, J: Parallel and Distributed Computation, Numerical Methods. Prentice-Hall, Englewood Cliff (1989)

16. Hoffmann, KH, Zou, J: Parallel algorithms of Schwarz variant for variational inequalities. Numer. Funct. Anal. Optim. 13 449-462 (1992)

17. Hoffmann, $\mathrm{KH}, \mathrm{Zou}, \mathrm{J}$ : Parallel solution of variational inequality problems with nonlinear source terms. IMA J. Numer. Anal. 16, 31-45 (1996)

10.1186/1029-242X-2013-509

Cite this article as: Guo et al.: A parallel resolvent method for solving a system of nonlinear mixed variational inequalities. Journal of Inequalities and Applications 2013, 2013:509

Submit your manuscript to a SpringerOpen ${ }^{\circ}$ journal and benefit from:

- Convenient online submission

- Rigorous peer review

- Immediate publication on acceptance

Open access: articles freely available online

- High visibility within the field

- Retaining the copyright to your article

Submit your next manuscript at $>$ springeropen.com 